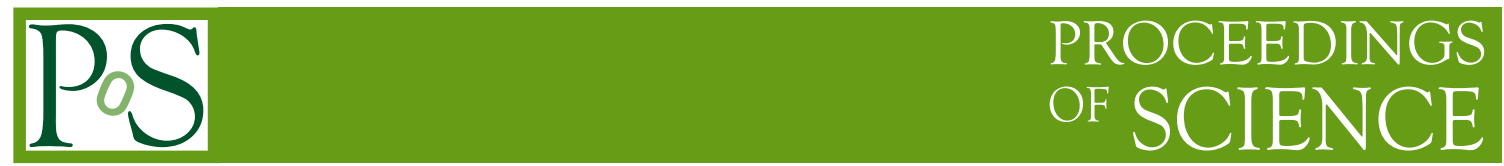

\title{
Top quark measurements
}

\author{
Alberto Orso Maria lorio, \\ Istituto Nazionale di Fisica Nucleare, sez. di Napoli, \\ for the ATLAS, CMS and LHCb collaborations.
}

Measurements of top quarks from Run-I and Run-II of the LHC are presented. Results on differential and inclusive top quark production cross sections, measured by the ATLAS, CMS and LHCb experiments, and measurements of top quark properties and mass are reported.

XIV International Workshop on Deep-Inelastic Scattering and Related Subjects

11-15 April, 2016

DESY Hamburg, Germany 


\section{Introduction}

Top quarks can be produced in pairs of top quark-antiquark (tî), mostly via strong interactions, or singly via electroweak interaction (single-top quark). Precision measurements in the top quark sector allow determination of fundamental standard model parameters, like the top quark mass and the Cabibbo-Kobayashi-Maskawa matrix element $V_{\mathrm{tb}}$, as well as measurements of parton distribution functions and detailed modeling of Quantum Chromodynamics (QCD) interactions. Properties of the top quark can be probed from its decay products, and allow the search to be performed for possible effects of physics beyond standard model affecting the production or decay vertices. All top quark processes constitute a background for beyond-standard-model physics searches.

\section{2. $\mathrm{t} \overline{\mathrm{t}}$ inclusive production}

The dominant production mechanism for top quark pairs at LHC is gluon-gluon fusion, which contributes for $85-90 \%$ depending on the centre-of-mass energy. $t \bar{t}$ production is a benchmark process for the standard model of particle physics, and precise measurement of its cross section allows for tests of perturbative QCD as well as of parton distribution functions (PDF). Top quark production is measured at ATLAS [1] and CMS [2] in central rapidity, and at LHCb [3] in more forward rapidity. For the most precise 7 and $8 \mathrm{TeV}$ measurements, ATLAS [4] defines collision events enriched in t⿱t by requring an electron-muon pair with opposite charge, plus one or two jets reconstructed as coming from b-quarks. The analysis from CMS [5] has a similar selection for what concerns leptons, but does not make any jet requirements. Instead a fit is performed to different event categories defined according to jet and b-jet multiplicity. The cross-sections for $t \bar{t}$ production at $\sqrt{s}=7,8$ are measured by the ATLAS and CMS collaborations to be:

$$
\begin{aligned}
& \sigma_{\mathrm{tt}}^{7 \mathrm{TeV}}=182.9 \pm 3.1(\text { stat }) \pm 4.2(\text { syst }) \pm 3.6(\text { lumi }) \pm 3.3(\text { beam }) \mathrm{pb}(\text { ATLAS }) \\
& \sigma_{\mathrm{tt}}^{7 \mathrm{TeV}}=173.6 \pm 2.1(\text { stat })_{-4.0}^{+4.5}(\text { syst }) \pm 3.8(\text { lumi }) \mathrm{pb}(\mathrm{CMS}), \\
& \sigma_{\mathrm{tt}}^{8 \mathrm{TeV}}=242.4 \pm 1.7(\text { stat }) \pm 5.5(\text { syst }) \pm 7.5(\text { lumi }) \pm 4.2(\text { beam }) \mathrm{pb}(\text { ATLAS }) \\
& \sigma_{\mathrm{tt}}^{8 \mathrm{TeV}}=244.9 \pm 1.4(\text { stat })_{-5.5}^{+6.3}(\text { syst }) \pm 6.4(\text { lumi }) \mathrm{pb}(\mathrm{CMS}) .
\end{aligned}
$$

For the latest measurements at $13 \mathrm{TeV}$, ATLAS [6] and CMS [7] obtain:

$$
\begin{aligned}
& \sigma_{\sigma_{\mathrm{t}}^{13 \mathrm{TeV}}}^{13 \mathrm{~T}}=818 \pm 8(\text { stat }) \pm 27(\text { syst }) \pm 19(\text { lumi }) \pm 12(\text { beam }) \mathrm{pb}(\text { ATLAS }) \\
& \sigma_{\mathrm{t} \mathfrak{\mathrm { t }}}^{13 \mathrm{TeV}}=793 \pm 8(\text { stat }) \pm 38(\text { syst }) \pm 21(\text { lumi }) \mathrm{pb}(\mathrm{CMS}) .
\end{aligned}
$$

For the latter result, CMS uses a cut-and-count approach rather than a maximum likelihood fit in order to extract the signal cross section. The distribution of the number of b-jets after the lepton selection is shown at $\sqrt{s}=13 \mathrm{TeV}$ in Fig. 1(a) for ATLAS and in Fig. 1(b) for CMS. The $\bar{t} \bar{t}$ cross-section evolution with the centre-of-mass energy is presented in Fig. 2.

The LHCb collaboration has observed top quark production at $\sqrt{s}=7$ and $8 \mathrm{TeV}$ [8] for the first time in forward rapidity pp collisions, obtaining a significance of 5.4 $\sigma$. Events with one muon 


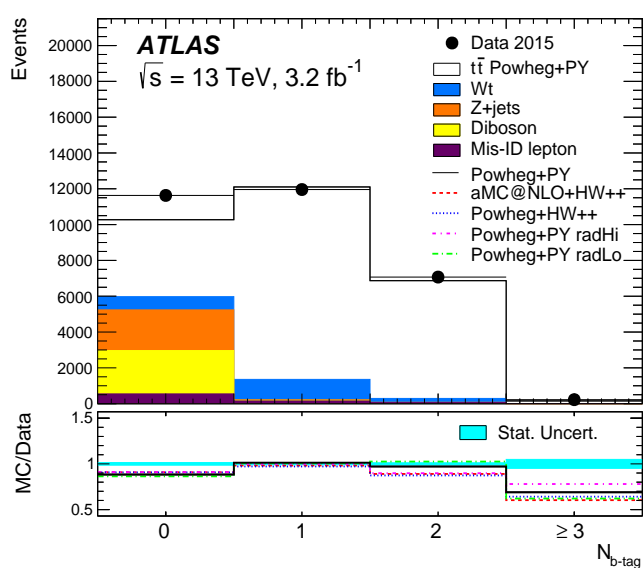

(a)

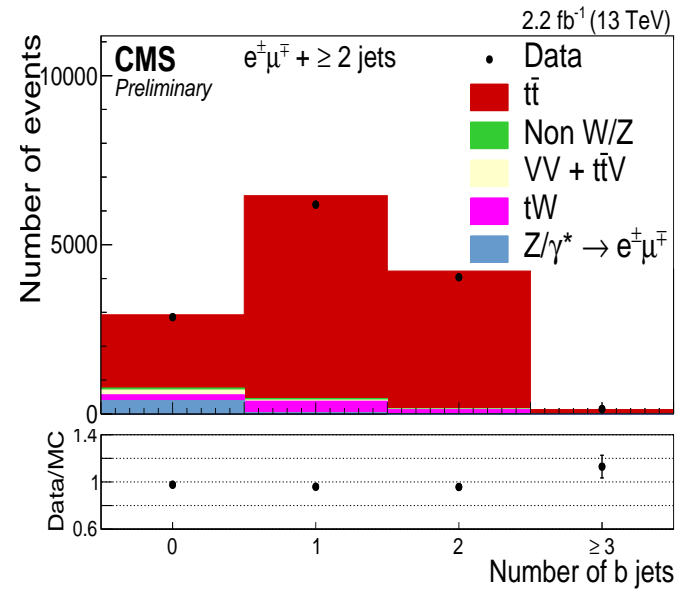

(b)

Figure 1: Distribution of the number of b-tagged jets in preselected opposite-sign $e \mu$ events compared to simulation for ATLAS(a) [6] and CMS(b) [7]

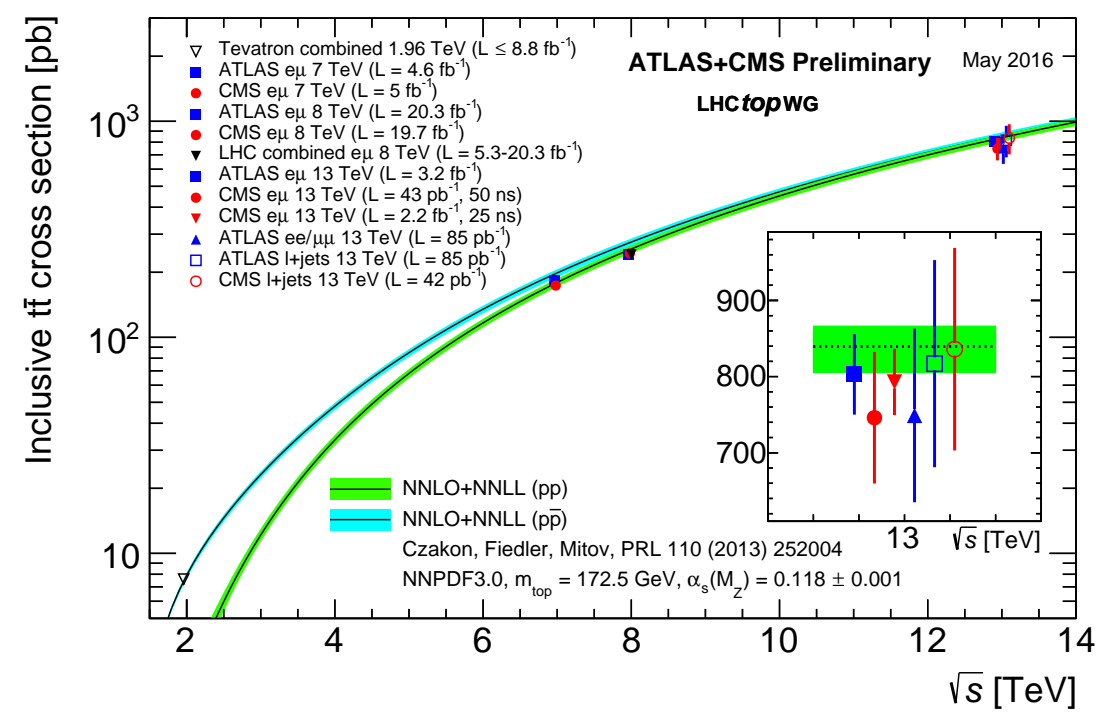

(a)

Figure 2: Cross-section for $\mathrm{t} \overline{\mathrm{t}}$ pair production in $p p$ collisions as a function of centre-of-mass energy compared to the NNLO+NNLL theoretical predictions for ATLAS and CMS [34].

$2<\eta<4.5$ and $p_{T}>25 \mathrm{GeV} / \mathrm{c}$, and one forward b-jet with $2.2<\eta<4.2$ and $25<p_{T}<100$ $\mathrm{GeV} / \mathrm{c}$ are selected. The top-quark cross section is determined by measuring the excess over $\mathrm{W}+\mathrm{b}$ jets background yield, obtaining:

$$
\begin{aligned}
& \sigma_{\mathrm{tt}}^{7 \mathrm{TeV}}=239 \pm 53 \text { (stat) } \pm 33 \text { (syst) } \pm 24 \text { (theory) fb }, \\
& \sigma_{\mathrm{t}}^{8 \mathrm{TeV}}=289 \pm 43 \text { (stat) } \pm 40 \text { (syst) } \pm 29 \text { (theory) fb. }
\end{aligned}
$$




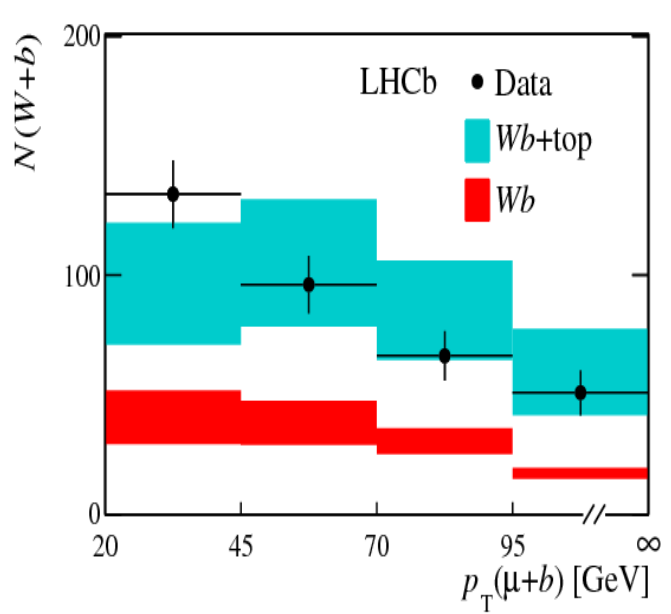

(a)

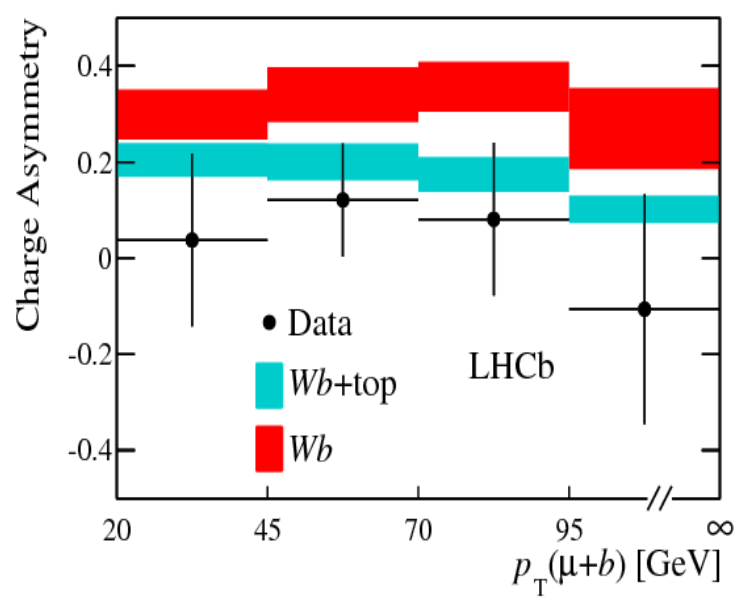

(b)

Figure 3: Distribution of the yield (a) and charge asymmetry (b) as function of the the $\mu$ and b-jet pair transverse momentum compared to SM predictions at NLO calculated with and without the contribution from top quark pair production [8].

\section{Associated production $t \bar{t}+$ jets}

The production of extra jet radiation in association with top quark pairs provides an important handle to study strong interactions. The normalised differential cross-sections of top-quark pair production as a function of the multiplicity of additional jets is measured by both ATLAS [9] and CMS [10][11] using the latest pp collision data at a centre-of-mass energy of $13 \mathrm{TeV}$ with a luminosity of $3.2 \mathrm{fb}^{-1}$ and $2.3 \mathrm{fb}^{-1}$ respectively. The measurements from both experiments are presented at particle-level fiducial phase space in order to reduce the model dependent uncertainties. CMS also extended the results at the parton level. ATLAS and CMS measure differential crosssections of top quark production as a function of addtional jet multuplicity, in the dilepton channel, in the ATLAS case, and in the dilepton and semi-leptonic channels for CMS. The cross section is normalised in order to exclude the uncertainties on the extrapolation from the total phase space to the observed phase space. The absolute differential cross section is measured by CMS, and found to be consistent with the standard model predictions. Some tension between data and prediction can be seen in the high jet multiplicity bins. Different tunes are used in ATLAS and CMS and that may reflect in opposite trends observed in each experiment, reported in Fig. 4.

\section{Differential ț production cross section}

The $t \bar{t}$ differential cross section at $13 \mathrm{TeV}$ with the 2015 data set was measured by CMS at $13 \mathrm{TeV}$, in both the semi-leptonic [10] and in the dilepton channel [11], in the first case selecting events with exactly one high energy lepton, at least four high transverse momentum jets, and at least one b-tagged jet, in the second selecting events with two leptons and at least two b-jets. The same measurement is performed by the ATLAS collaboration at $8 \mathrm{TeV}$ [12]. The event selection and analysis strategy is similar for the two cases, allowing for independent but comparable results, shown in Fig. 5. For both analyses, MC predictions overestimate what is found in data, especially 


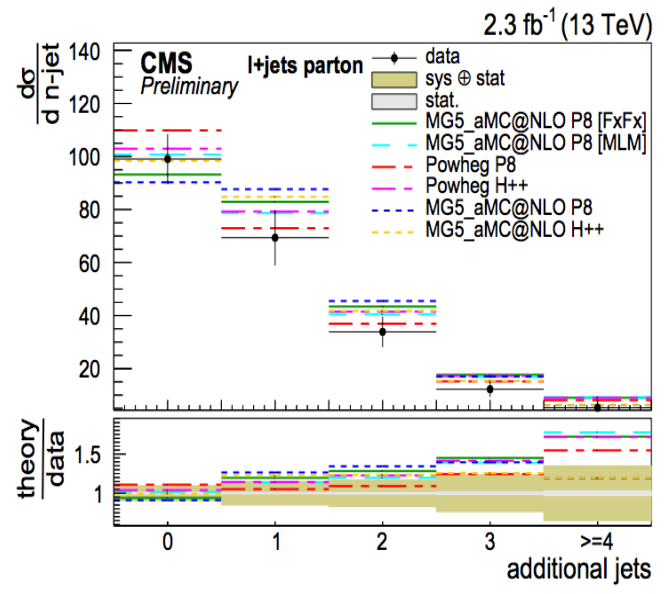

(a)

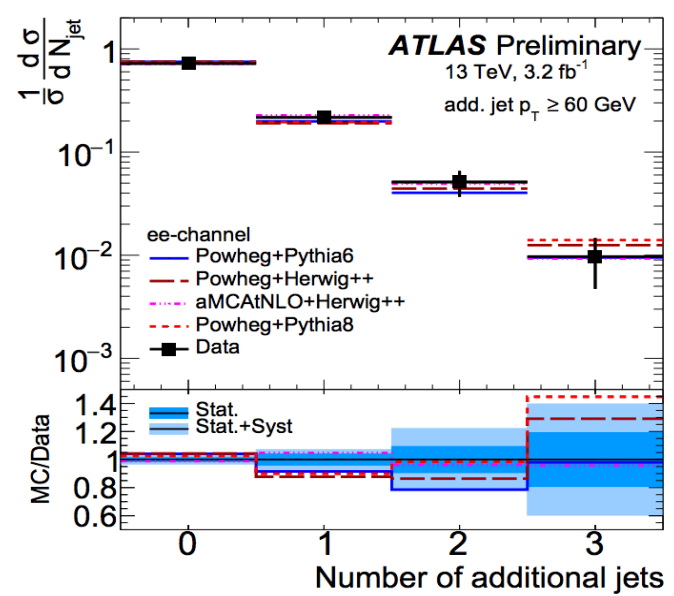

(b)

Figure 4: $t \bar{t}$ production cross section as a function of the number of additional jets from CMS (a) [10] and ATLAS (b) [9].

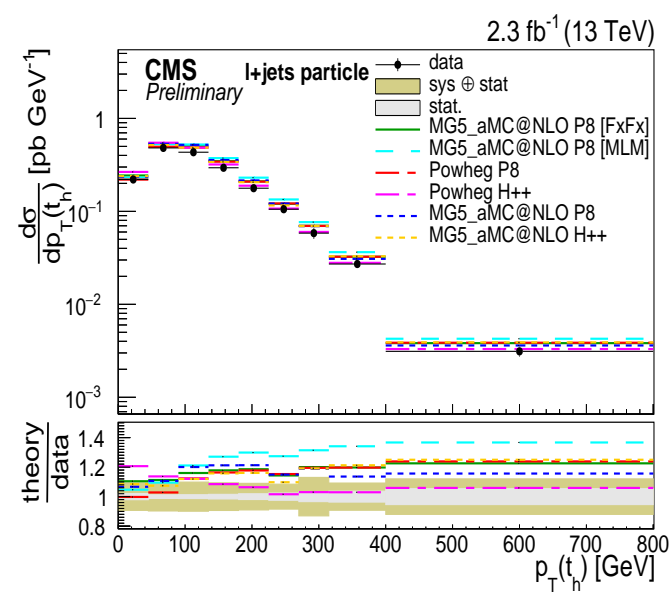

(a)

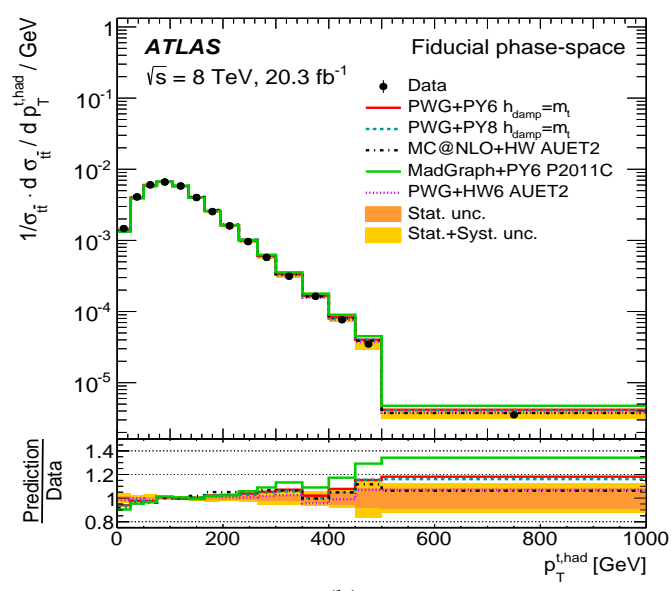

(b)

Figure 5: $\mathrm{t} \overline{\mathrm{t}}$ production cross section as a function of the hadronic top quark $p_{T}$ in the boosted regime from CMS at $13 \mathrm{TeV}(\mathrm{a})[10]$ and ATLAS at $8 \mathrm{TeV}(\mathrm{b})[12]$.

at high energies. A better agreement is found when using the new NNLO QCD predictions [13, 14].

\section{5. $t \bar{t}$ charge asymmetry}

A potential deviation from the standard model could manifest as an asymmetry in the rapidity distribution of top quarks with respect to antiquarks. The measurement of the asymmetry, defined as:

$$
A_{C}=\frac{N(\Delta|y|>0)-N(\Delta|y|<0)}{N(\Delta|y|>0)+N(\Delta|y|<0)}
$$

is performed at both the ATLAS [15][16] and CMS [17] experiments with LHC data at $8 \mathrm{TeV}$. The analyses are performed in the lepton-plus-jets channel and in both the resolved and boosted 
kinematic regime. All results are compatible with the standard model predictions within in two

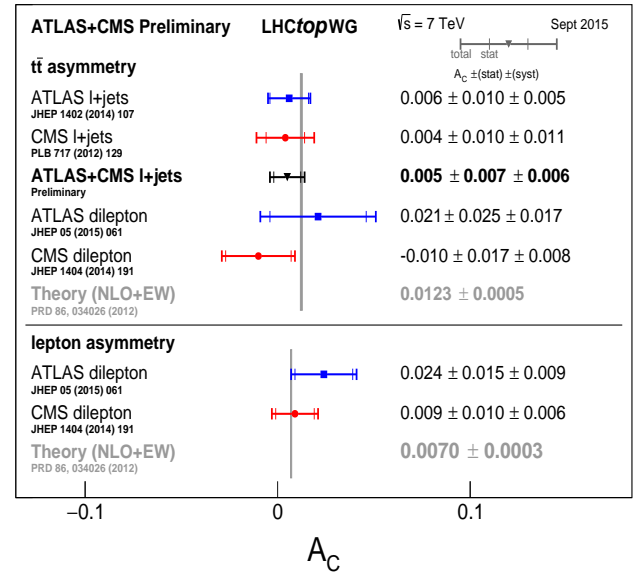

(a)

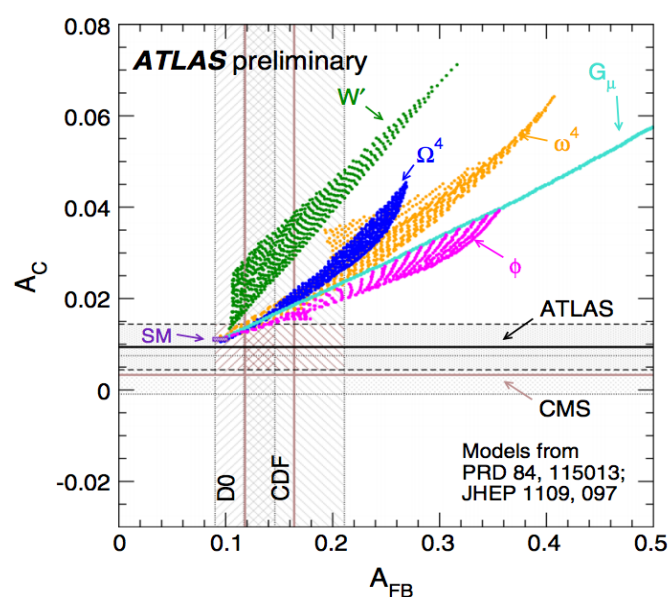

(b)

Figure 6: (a) Charge asymmetry observable $A_{C}$ measured at $7 \mathrm{TeV}$ in ATLAS and CMS and (b) observed charged asymmetry comared with SM and BSM predictions [15].

standard deviations, as shown in Fig. 6(a), and provide constraints on several extensions of the SM, as shown in Fig. 6(b).

\section{Top quark polarization}

Measurements of angular properties of top quark decay products might indicate the presence of anomalous spin correlations amongst the top quarks in production, or a top quark polarisation[18][19]. Different observables are used to probe top quark polarisation and spin correlation, depending also on the decay channel of the top quarks: the angle $\theta_{l} *$ between the lepton and its originating top quark in the top rest frame is used in semileptonic $t \bar{t}$ decay channels, while the $\phi$ angle between the two leptons is used for the dilepton $\mathrm{t} \overline{\mathrm{t}}$ decay channels. The measured top quark polarization and the spin correlation observables are compared to theoretical predictions in Fig. 7, allowing to set constraints to beyond SM theories. Top quark polarisation has been probed in single-top-quark events as well by CMS [20].

\section{Single top quarks}

Single-top-quark production happens via electroweak charged-current interaction, through virtual exchange of $\mathrm{W}$ bosons in the $t, s$ channels or via associated production with a real $\mathrm{W}$ boson (tW). All three channels allow measurement to be made of the modulus squared of the CabibboKobayashi-Maskawa matrix element $V_{\mathrm{tb}}$, and are sensitive to anomalous couplings in the tWb vertex, providing a complementary route to study top quark properties with respect to strong tt production. Single-top-quark cross-section measurements at LHC are summarized in Fig 8(a), while $\left|V_{\mathrm{tb}}\right|$ measurements are shown in Fig. 8(b).

The single-top-quark $t$ channel production is the most abundant, and precise measurements were performed at $7[21,22], 8[23,24]$, and $13[25,26] \mathrm{TeV}$ by both the ATLAS and CMS collaborations, resulting in: 


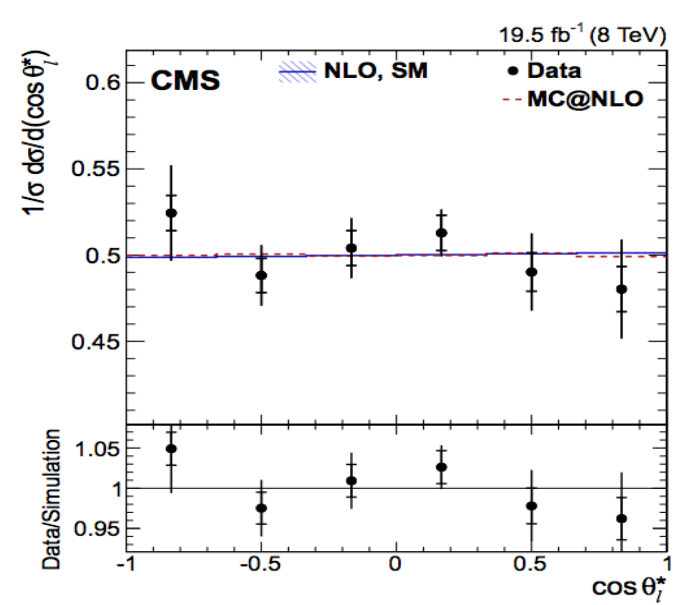

(a)

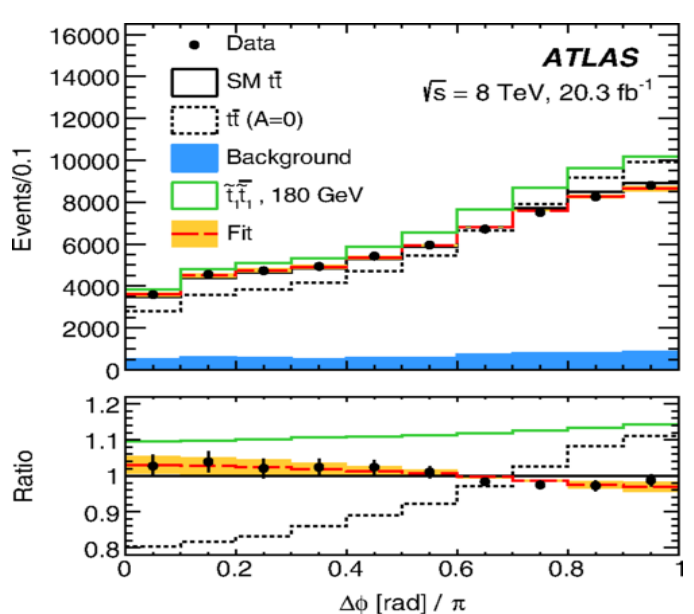

(b)

Figure 7: Distributions of the top polarization angles $\cos (\theta *)$ (a) [19] and $\phi_{l}$ (b) [18].

$$
\begin{aligned}
& \sigma_{t-\mathrm{ch} .}^{7 \mathrm{TeV}}=68.2 \pm 2(\text { stat }) \pm 8(\text { syst }+ \text { lumi }) \mathrm{pb}(\text { ATLAS }), \\
& \sigma_{t-\text { ch. }}^{7 \mathrm{TeV}}=67.2 \pm 3.7(\text { stat }) \pm 4.6(\text { syst }) \pm 1.5(\text { lumi }) \mathrm{pb}(\mathrm{CMS}), \\
& \sigma_{t-\mathrm{ch} .}^{8 \mathrm{TeV}}=82.6 \pm 1.2(\text { stat }) \pm 11.8(\text { syst }) \pm 2.3(\text { lumi }) \mathrm{pb}(\text { ATLAS }), \\
& \sigma_{t-\mathrm{ch} .}^{8 \mathrm{TeV}}=87.2 \pm 2.3(\text { stat }) \pm 7.4(\text { syst }+ \text { lumi }) \mathrm{pb}(\mathrm{CMS}) .
\end{aligned}
$$

for the Run-I measurements, and for the Run-II at $13 \mathrm{TeV}$ :

$$
\begin{aligned}
& \sigma_{t-\text { ch. }}^{13 \mathrm{TeV}}=229 \pm 48(\text { stat }+ \text { syst }+ \text { lumi }) \mathrm{pb}(\text { ATLAS }), \\
& \sigma_{t-\text { ch. }}^{13 \mathrm{TeV}}=227.8 \pm 9.1(\text { stat }) \pm 31.5(\text { syst }) \pm 6.2(\text { lumi }) \mathrm{pb}(\mathrm{CMS}) .
\end{aligned}
$$

The $13 \mathrm{TeV}$ analyses make use of multivariate discriminants to separate the $t$ channel signal from the backgrounds, shown in Fig. 9(a), 9(b) for ATLAS and CMS respectively.

Another peculiar feature of $t$ channel production is the difference in production cross section of top quark over top antiquark, which stems from the $\mathrm{u}$ and $\mathrm{d}$ parton distribution functions. This asymmetry can be used as a handle in the PDF fits. The measurements of $\sigma_{t-\text { ch.,t }} / \sigma_{t-\mathrm{ch.t}, \mathrm{t}}$, also called $R_{t / \bar{t}}$, at 7,8, and $13 \mathrm{TeV}$ are compared with different PDF prediction in Fig. 10(a), 10(b), and 10 (c) for the three energies $[22,23,25]$. The production of single top quarks in association with W bosons was first observed in 2014 by CMS [27], and subsequently by ATLAS in 2015 [28], in events with two leptons, either electrons or muons. Multivariate discriminants, displayed in Fig. 11, are used in both analyses to discriminate signal from backgrounds, yielding measured tW cross sections at $8 \mathrm{TeV}$ of $23.0 \pm 3.6 \mathrm{pb}$ (ATLAS) and 23.4 \pm 5.4 (CMS) pb. 


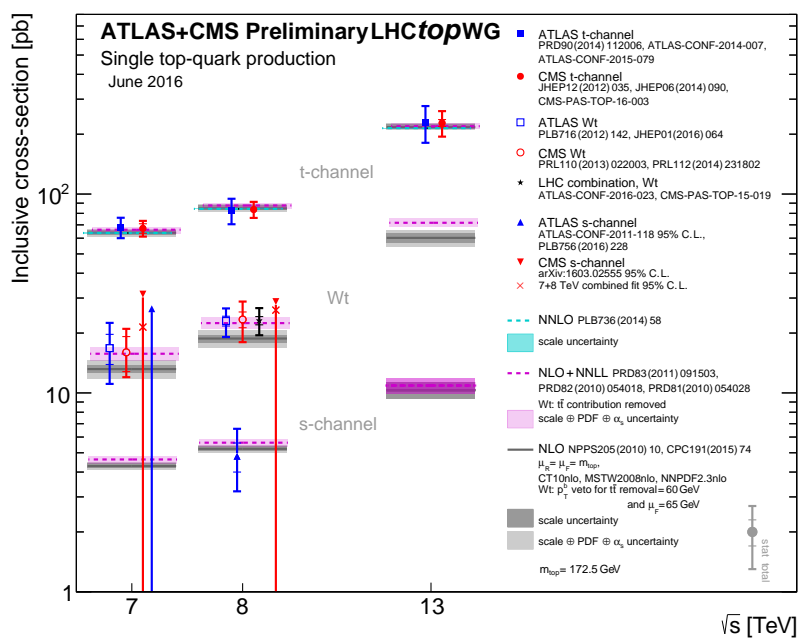

(a)

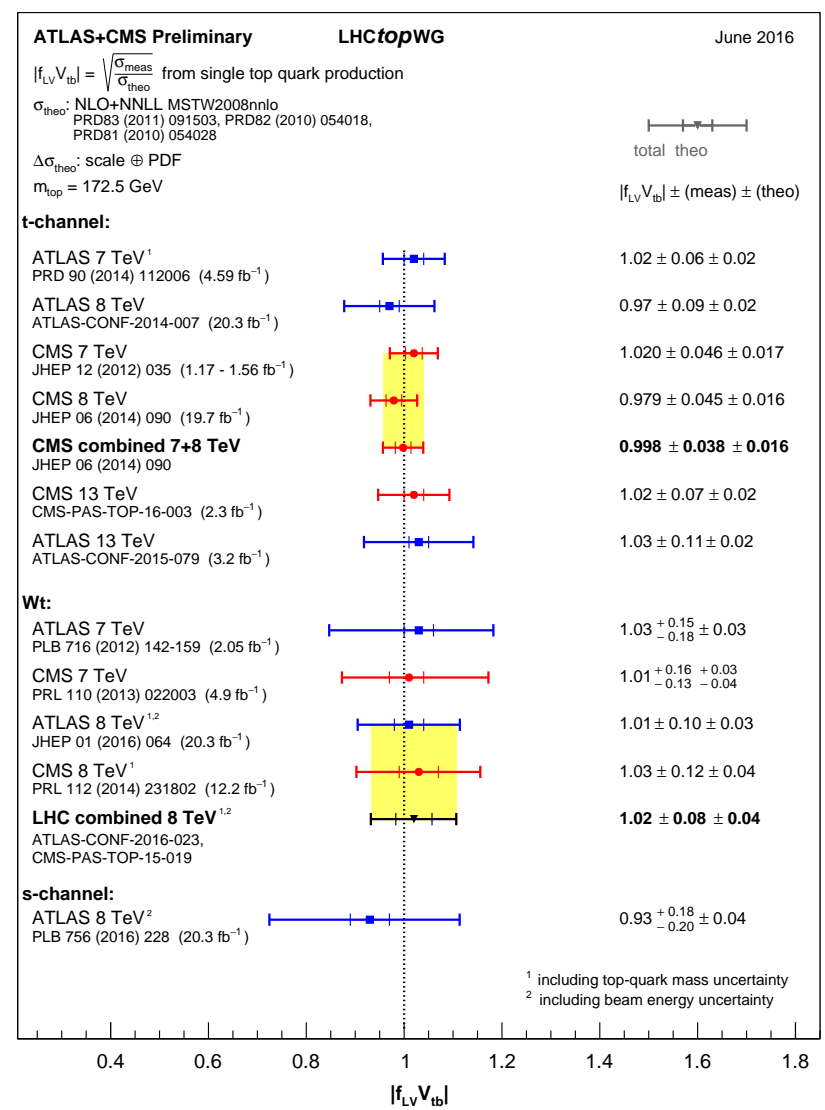

(b)

Figure 8: Single-top-quark production cross-section measurements at LHC as a function of the centre-ofmass energy(a) and measurements of $\left|V_{\mathrm{tb}}\right|$ from inclusive single-top-quark cross section(b) [34].

$$
\begin{aligned}
& \sigma_{s-\text { ch. }}^{8 \mathrm{TeV}}=4.8 \pm 0.8(\text { stat })_{-1.3}^{+1.6}(\text { syst }) \mathrm{pb}(\text { ATLAS }), \\
& \sigma_{s-\text { ch. }}^{8 \mathrm{TeV}}=13.4 \pm 7.3(\text { stat }+ \text { syst }) \mathrm{pb}(\mathrm{CMS}), \\
& \sigma_{s-\text { ch. }}^{7 \mathrm{TeV}}=7.1 \pm 8.1(\text { stat }+ \text { syst }) \mathrm{pb}(\mathrm{CMS}) .
\end{aligned}
$$




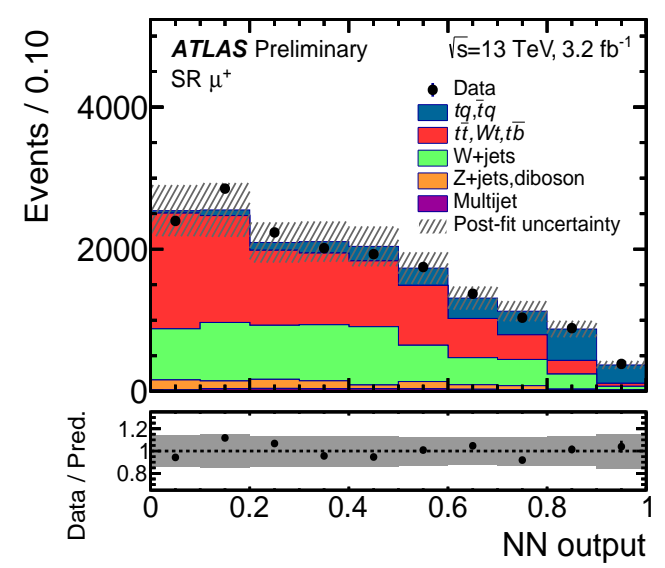

(a)

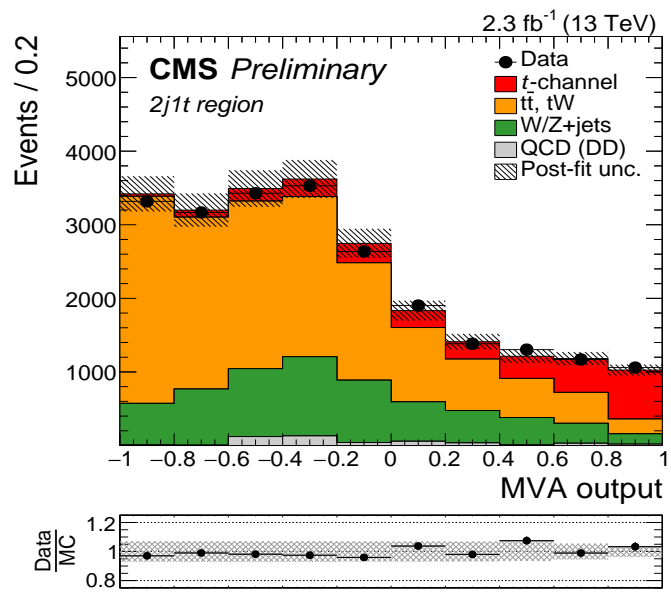

(b)

Figure 9: Discriminating observable used for $t$ channel single-top-quark production cross section extraction for ATLAS, the output discriminator of a boosted decision tree Method(a) [26], and for CMS, the output discriminator of a neural network(b) [25], at $13 \mathrm{TeV}$.

\section{Top quark mass measurements}

The mass of the top quark is one of the fundamental parameters of the standard model and is measured with high precision from top quark decay products. The most precise single measurements come from the LHC Run-I at $8 \mathrm{TeV}$, profiting from the latest detector calibrations. The most precise CMS measurement[31] exploits the semi-leptonic decay channel, requiring one lepton and at least four jets, hadronically decaying top quark from three jets. A kinematic fit is performed to each jet permutation forming the hadronically decaying top quark. The goodness of fit probability for all possible permutations is used to construct an event-by-event likelihood and to measure the top quark mass. The most precise ATLAS[32] measurement exploits the dileptonic decay channel, requiring two leptons and two b-jets, retaining the permutation with the lowest invariant mass possible of the two lepton-b-jet pair. Both ATLAS and CMS extract simultaneously the top quark mass together with the jet energy scale from $t \bar{t}$. The resulting measured top quark mass is for the two cases:

$$
\begin{aligned}
& m_{\text {top }}=172.99 \pm 0.41(\mathrm{stat}) \pm 0.74(\mathrm{syst}) \mathrm{GeV} / \mathrm{c}^{2}(\text { ATLAS }), \\
& m_{\text {top }}=172.35 \pm 0.16(\mathrm{stat}+\mathrm{jsf}) \pm 0.48(\mathrm{syst}) \mathrm{GeV} / \mathrm{c}^{2},(\mathrm{CMS}) .
\end{aligned}
$$

Fig. 13(a), 13(b), show the observables used for the top quark mass extraction respectively for ATLAS and CMS. Top quark mass measurements with different techniques were performed to improve on the systematic uncertainty. The LHCTopWG has performed a combination of measurements from the LHC and Tevatron experiments [33], and an overview of the LHC measurements is shown in Fig. 14. 


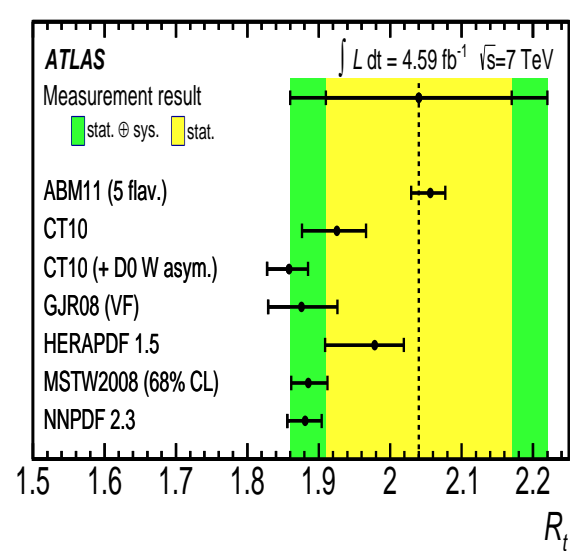

(a)

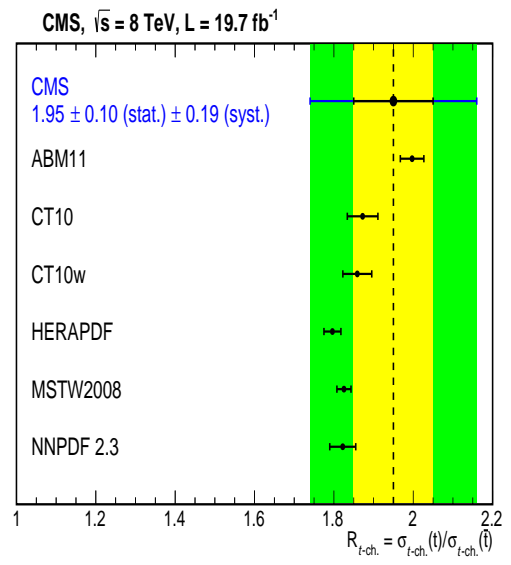

(b)

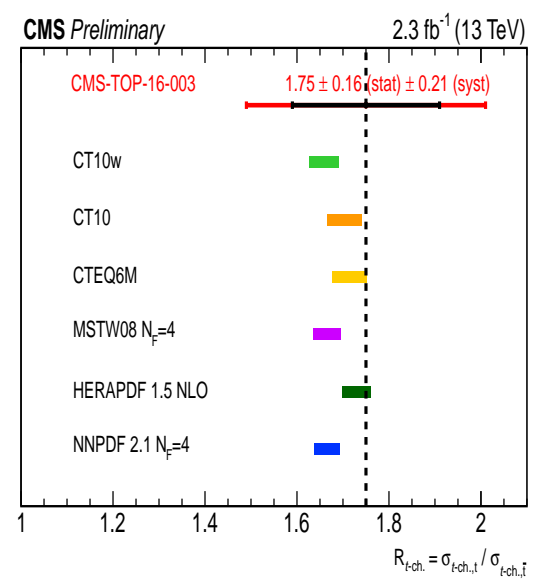

(c)

Figure 10: Ratio of top quark over top antiquark $t$-channel cross section at $7 \mathrm{TeV}$ for ATLAS(a) [22], at 8 $\mathrm{TeV}$ for CMS(b) [21], and at $13 \mathrm{TeV}$ for CMS(c) [25].

\section{Conclusions}

Measurements of the inclusive cross sections for production at the LHC of top quarks, produced either singly or in pairs, have been presented for 7,8 and $13 \mathrm{TeV}$ centre of mass energies. All cross sections confirm the standard model predictions. Differential production cross sections of top quark pairs, measured as a function of additional jet multiplicity as well as of top quark pair kinematic properties, have been displayed, allowing the modeling of Quantum Chromodynamics interactions to be probed in detail. Measurements of top quark properties, sensitive to potential beyond standard model physics, have been reported, and no evidence of such models was found. The most precise measurements of top mass have also been shown.

\section{References}

[1] G. Aad et al. [ATLAS Collaboration], JINST, 3 (2008) S08003 


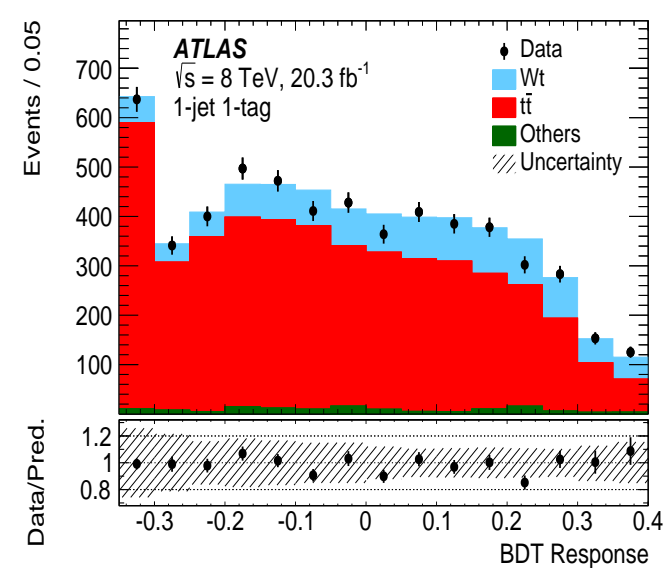

(a)

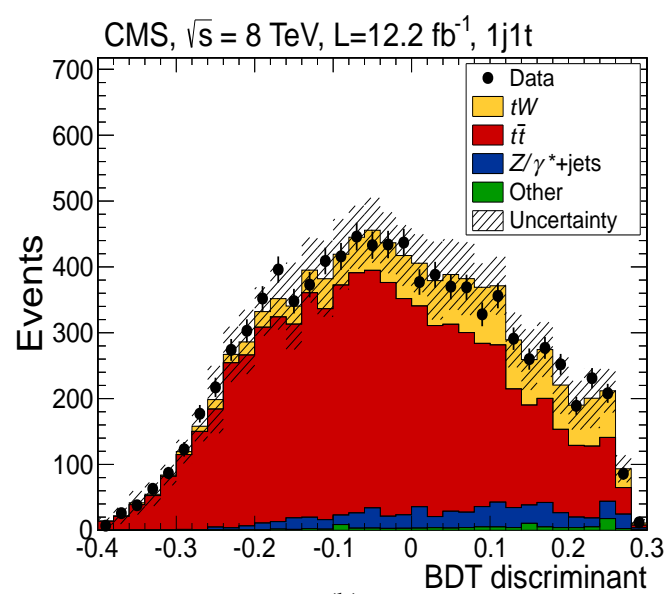

(b)

Figure 11: Discriminating observable used for $\mathrm{W}$ associated single-top-quark production cross section extraction for CMS(a) [27] and ATLAS(b) [28] at $8 \mathrm{TeV}$ ). In both cases it is the output discriminator of a boosted decision tree.

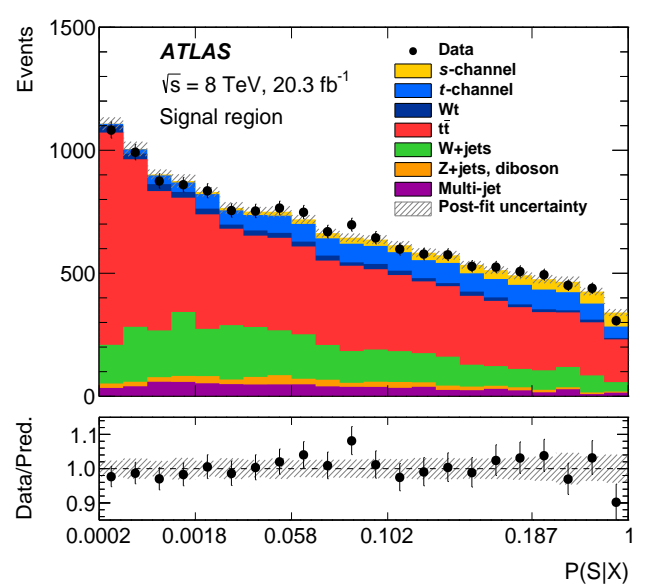

(a)

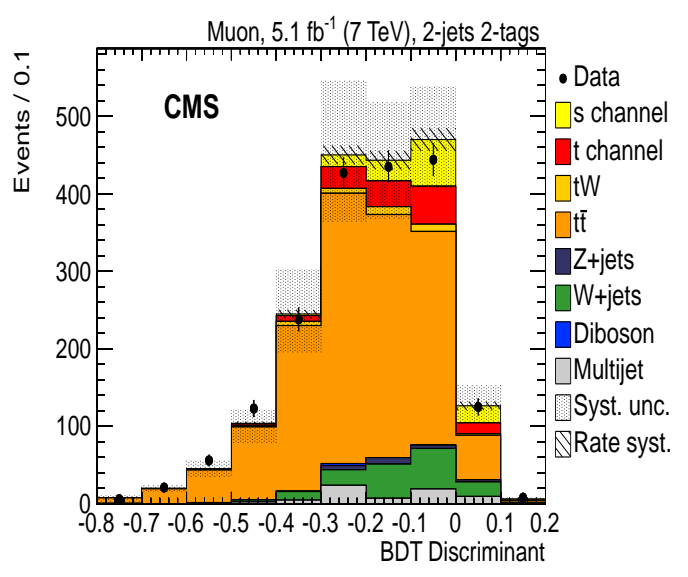

(b)

Figure 12: Discriminating observable used for $s$ channel single-top-quark production cross section extraction for ATLAS at $8 \mathrm{TeV}$, a Matrix Element Method output discriminator(a) [29], and CMS at $7 \mathrm{TeV}$, a boosted decision tree output discriminator(b) [30].

[2] V. Khachatryan et al. [CMS Collaboration], JINST, 3 (2008) S08004

[3] R. Aaij et al. [LHCb Collaboration], JINST, 3 (2008) S08005

[4] G. Aad et al. [ATLAS Collaboration], Eur. Phys. J. C 74, no. 10, 3109 (2014) doi:10.1140/epjc/s10052-014-3109-7 [arXiv:1406.5375 [hep-ex]].

[5] V. Khachatryan et al. [CMS Collaboration], [arXiv:1603.02303 [hep-ex]].

[6] M. Aaboud et al. [ATLAS Collaboration], Phys. Lett. B 761 (2016) 136, doi:10.1016/j.physletb.2016.08.019. [arXiv:1606.02699 [hep-ex]].

[7] V. Khachatryan et al. [CMS Collaboration], CMS-PAS-TOP-16-005. 


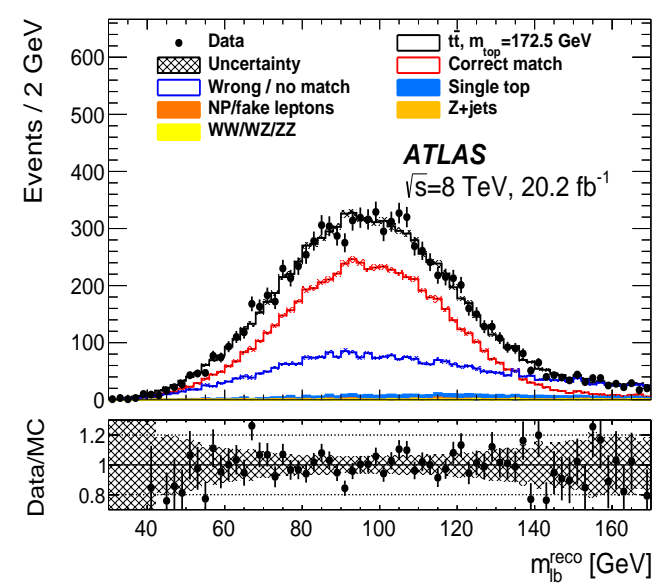

(a)

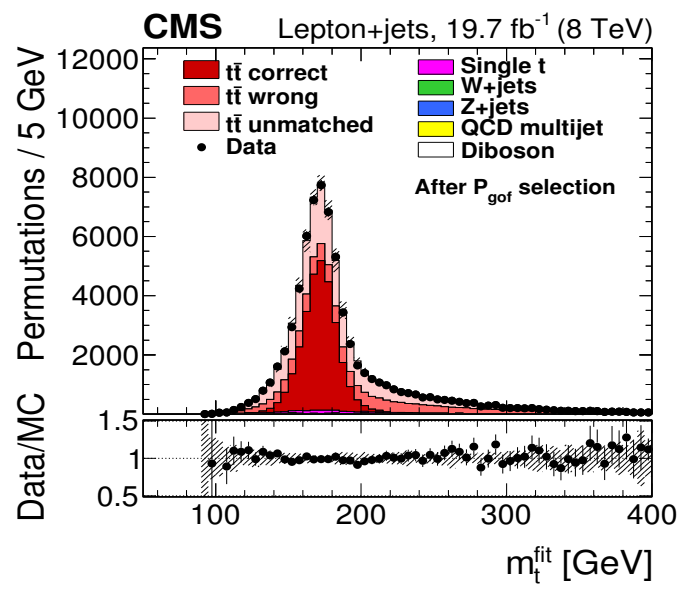

(b)

Figure 13: Mass of the lepton-b-jet pair from ATLAS [32], mass of the three jets from the best permutation from CMS [31].

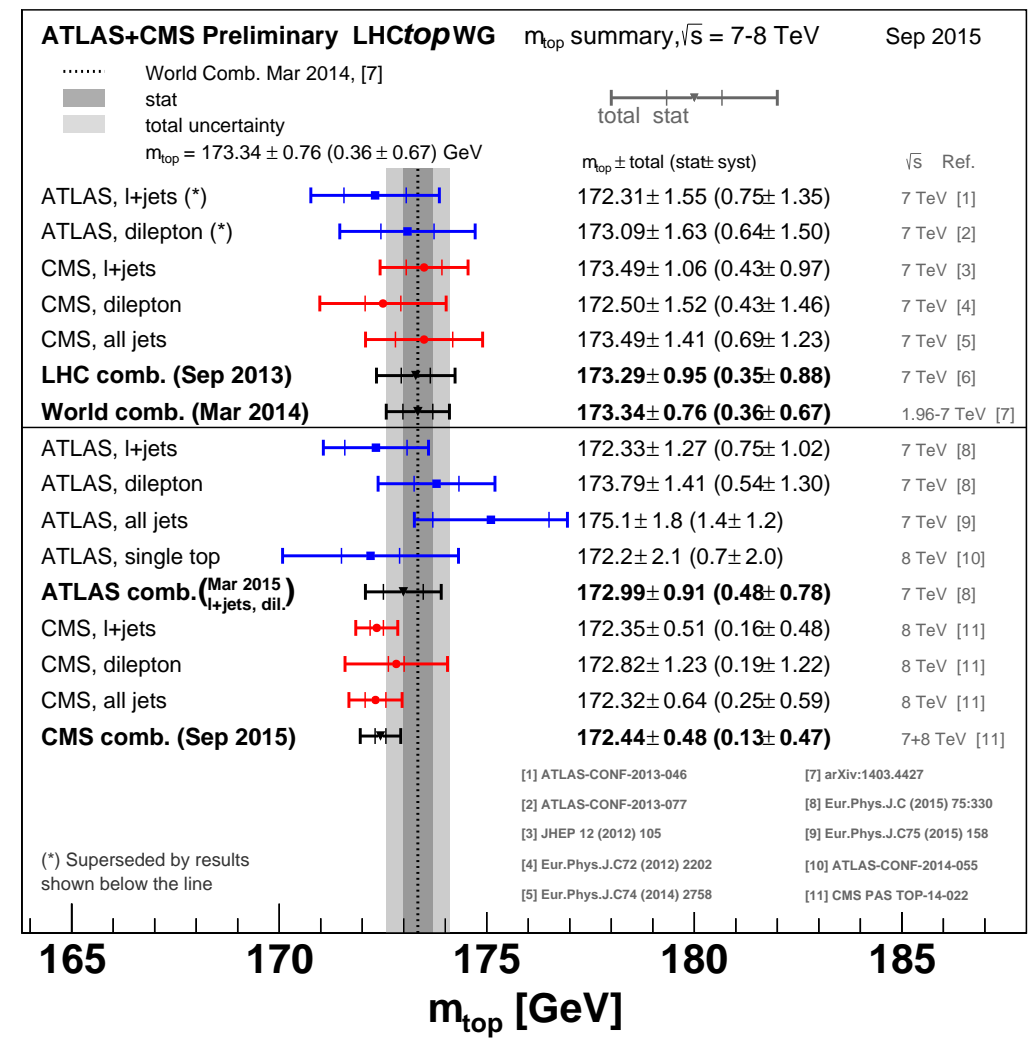

(a)

Figure 14: Overview of the most precise single measurements of the top quark mass and top quark mass combinations [34].

[8] R. Aaij et al., [LHCb Collaboration], Phys. Rev. Lett. 115, no. 11, (2015) 112001 doi:10.1103/PhysRevLett.115.112001 [arXiv:1506.00903 [hep-ex]]. 
[9] G. Aad et al. [ATLAS Collaboration], ATLAS-CONF-2015-065.

[10] V. Khachatryan et al. [CMS Collaboration], CMS-PAS-TOP-16-008.

[11] V. Khachatryan et al. [CMS Collaboration], CMS-PAS-TOP-16-011.

[12] G. Aad et al. [ATLAS Collaboration], arXiv:1511.04716[hep-ex].

[13] N. Kidonakis, Phys. Rev. D 91 (2015) 031501.

[14] B.D. Pecjak et al. Phys. Rev. Lett. 116 (2016 ) 202001,

[15] G. Aad et al. [ATLAS Collaboration], Eur. Phys. J. C 76 (2016) no.2, 87 doi:10.1140/epjc/s10052-016-3910-6 [arXiv:1509.02358 [hep-ex]].

[16] G. Aad et al. [ATLAS Collaboration], Phys. Lett. B 756 (2016) 52 doi:10.1016/j.physletb.2016.02.055 [arXiv:1512.06092 [hep-ex]].

[17] V. Khachatryan et al. [CMS Collaboration], Phys. Lett. B 757 (2016) 154 doi:10.1016/j.physletb.2016.03.060 [arXiv:1507.03119 [hep-ex]].

[18] G. Aad et al. [ATLAS Collaboration], Phys. Rev. Lett. 114, no 14, 142001 (2015) doi:10.1103/PhysRevLett.114.142001

[19] V. Khachatryan et al. [CMS Collaboration], Phys. Lett. D 93 (2016) 052007 doi:10.1103/PhysRevD.93.052007

[20] V. Khachatryan et al. [CMS Collaboration], [CMS Collaboration], JHEP 04 (2016) 073.

[21] V. Khachatryan et al. [CMS Collaboration], JHEP 12 (2012) 035.

[22] G. Aad et al. [ATLAS Collaboration], Phys. Rev. D 90 (2014) 112006.

[23] V. Khachatryan et al. [CMS Collaboration], JHEP 06 (2014) 090.

[24] G. Aad et al. [ATLAS Collaboration], ATLAS-CONF-2014-007.

[25] V. Khachatryan et al. [CMS Collaboration], CMS-PAS-TOP-16-003.

[26] G. Aad et al. [ATLAS Collaboration], ATLAS-CONF-2015-079.

[27] V. Khachatryan et al. [CMS Collaboration], Phys. Rev. Lett. 112 (2014) 231802.

[28] G. Aad et al. [ ATLAS Collaboration], JHEP 01 (2016) 064.

[29] G. Aad et al. [ ATLAS Collaboration], Phys.Lett.B 756 (2016) 228-246.

[30] V. Khachatryan et al. [CMS Collaboration], JHEP 09 (2016) 027.

[31] V. Khachatryan et al. [CMS Collaboration], Phys. Rev. D 93 (2016) 072004.

[32] G. Aad et al. [ ATLAS Collaboration], Phys.Lett.B 761 (2016) 350-371.

[33] The ATLAS, CMS, CDF, and D0 Collaborations, arXiv:1403.4427.

[34] The LHC Top Working group, LHCTopWG summary plots, https://twiki.cern.ch/twiki/bin/view/LHCPhysics/LHCTopWG 